\title{
Conversion therapy for gastric cancer: who can make conversion as successful as Goromaru?
}

\author{
Masanori Terashima ${ }^{1}$
}

Received: 28 February 2016/ Accepted: 30 March 2016/Published online: 7 April 2016

(c) The International Gastric Cancer Association and The Japanese Gastric Cancer Association 2016

Conversion therapy (surgery) is the case where the surgical resection is determined to be inapplicable to a tumor, and later, usually after the chemotherapy, surgical resection is performed. This practice is adopted to achieve a radical cure and therefore, differs from salvage surgery. To date, chemotherapy has had limited effects on gastrointestinal cancer, which is why, in most cases, resection after chemotherapy is performed as palliative treatment for stenosis, bleeding, and other complications. However, since the early 2000s, huge advances in chemotherapy have been made in the field of colorectal cancer, which was previously thought to be treatment resistant. Resection is now actively performed following chemotherapy, particularly in cases of liver metastasis [1]. Conversion therapy is currently recognized as a significant factor for improving life expectancy in cases of advanced and recurrent colorectal cancer.

However, there are few reports on conversion surgery in cases of gastric cancer-only a few case series from single facilities have been published in Japan and South Korea.

Nakajima et al. [2] at the Cancer Institute Hospital in Japan were the first to report on conversion surgery for gastric cancer. In 1997, they reported on 30 initially incurable patients who underwent surgeries following two cycles of FLEP (5-FU, leucovorin, cisplatin, etoposide, i.v. and i.a.) therapy. A response to FLEP therapy was seen in $50 \%$ of the patients, and 19 patients underwent surgery.

This comment refers to the article available at doi:10.1007/s10120015-0575-z.

Masanori Terashima

m.terashima@scchr.jp

1 Division of Gastric Surgery, Shizuoka Cancer Center, Nagaizumi-Cho, Shizuoka, Japan
Curative resection was achieved in nine $(47 \%)$ of the patients with locally advanced cancer or lymph node metastasis. Nakajima et al. also reported that control was difficult in patients with liver metastasis and/or peritoneal seeding and that a 5-year survival rate of $55.6 \%$ was achieved in cases of lymph node metastasis that were curatively resected. Because resection was planned from the start and the number of cycles of chemotherapy was pre-determined, this report is the first to present cases of post-chemotherapy resection that were initially unresectable, despite the fact that the current implications for therapy differ slightly. While the term "unresectable" is used, the situation is actually more varied, and it has only recently been suggested that cases of $\mathrm{T} 4 \mathrm{~b}$ cancer and distant lymph node metastasis are good candidates for conversion.

Thereafter, in 2002, Yano et al. [3] from Osaka University reported on surgical procedures that were performed in initially unresectable cases of gastric cancer, which were judged to be resectable after FEMTXP (5fluorouracil, epirubicin, methotrexate, cisplatin) or THPFLPM (pirarubicin, 5-fluorouracil, leucovorin, cisplatin, mitomycin C) regimens. While Yano et al. used the term "salvage surgery," they most likely used a strategy that corresponded to the current concept of conversion therapy. Fourteen out of 33 patients underwent surgeries, and curative resections were achieved in 8 of them. Analysis of the prognostic factors revealed that resectability was the sole independent prognostic factor.

Yoshida et al. [4] presented the concept of "adjuvant surgery," in which resection is performed after a response to chemotherapy-a concept that is now synonymous with the current term of conversion therapy. From among 20 cases of adjuvant surgery, they reported good survival in 
cases with a response to chemotherapy and curative resection but poor survival in cases of peritoneal seeding.

Subsequent outcomes of conversion therapy have also been reported, primarily in Japan [5-8], and curative resection has been reported as an independent prognostic factor in all of these cases. In a study of curative resection cases, the tendency for good survival was seen in cases with strong histological effects. Kanda et al. [9] also reported that histological tumor diameter was an independent prognostic factor. Lymph node metastasis was found to be an initial non-curable factor with the best outcomes, whereas conversion from peritoneal seeding was considered to have poor outcomes. Kim et al. [10] reported outcomes of conversion surgery for peritoneal seeding, but surgery was only possible in 18 out of 43 cases, of which curative resection was possible in only 10 . However, the 3 -year survival rate for curatively resectable cases was $50 \%$.

Thus, while relatively good outcomes have been reported for conversion surgery, the criteria for initial determination of "non-curative" or determination of resectability post-chemotherapy have not been established; they differ greatly from facility to facility. In a recently reported clinical trial, only 5 out of 86 patients $(6 \%)$ underwent conversion surgery [11].

Randomized controlled trials are the ultimate means of evaluating the efficacy of conversion surgery; however, the presence of wide variations in the examined background factors makes conducting randomized controlled trials extremely difficult in practice. To establish evidence, prospective cohort studies may need to be conducted. However, because conversion therapy is not recognized internationally, for the time being, it is important to establish a common understanding of the term by raising awareness of conversion therapy. Based on biological characteristics, Yoshida et al. [12] classified stage IV cases into four categories. According to them, the most important category is peritoneal seeding, and marginal resectable tumors without peritoneal seeding are the best candidates for conversion therapy. On the basis of this classification, a joint international cohort study is planned in Asia, which should represent a huge step forward in establishing evidence for conversion therapy.

Conversion surgery can also signify additional benefits, just like a conversion after a touchdown in football or a try in rugby. We want to make "conversion" as successful in the treatment of gastric cancer as a precise kick by Goromaru.

\section{References}

1. Adam R, Delvart V, Pascal G, Valeanu A, Castaing D, Azoulay $\mathrm{D}$, et al. Rescue surgery for unresectable colorectal liver metastases downstaged by chemotherapy: a model to predict long-term survival. Ann Surg. 2004;240:644-57.

2. Nakajima T, Ota K, Ishihara S, Oyama S, Nishi M, Ohashi Y, Yanagisawa A. Combined intensive chemotherapy and radical surgery for incurable gastric cancer. Ann Surg Oncol. 1997;4:203-8.

3. Yano M, Shiozaki H, Inoue M, Tamura S, Doki Y, Yasuda T, et al. Neoadjuvant chemotherapy followed by salvage surgery: effect on survival of patients with primary noncurative gastric cancer. World J Surg. 2002;26:1155-9.

4. Suzuki T, Tanabe K, Taomoto J, Yamamoto H, Tokumoto N, Yoshida K, Ohdan H. Preliminary trial of adjuvant surgery for advanced gastric cancer. Oncol Lett. 2010;1:743-7.

5. Ishigami S, Natsugoe S, Nakajo A, Matsumoto M, Uenosono Y, Arigami $\mathrm{T}$, et al. Salvage gastrectomy following a combination of biweekly paclitaxel and S-1 for stage IV gastric cancer. J Gastrointest Surg. 2008;12:1370-5.

6. Satoh S, Okabe H, Teramukai S, Hasegawa S, Ozaki N, Ueda ST, et al. Phase II trial of combined treatment consisting of preoperative S-1 plus cisplatin followed by gastrectomy and postoperative S-1 for stage IV gastric cancer. Gastric Cancer. 2012;15:61-9.

7. Aizawa M, Nashimoto A, Yabusaki H, Nakagawa S, Matsuki A, Homma K, Kawasaki T. The clinical significance of potentially curative resection for gastric cancer following the clearance of free cancer cells in the peritoneal cavity by induction chemotherapy. Surg Today. 2015;45:611-7.

8. Fukuchi M, Ishiguro T, Ogata K, Suzuki O, Kumagai Y, Ishibashi $\mathrm{K}$, et al. Prognostic role of conversion surgery for unresectable gastric cancer. Ann Surg Oncol. 2015;22:3618-24.

9. Kanda T, Yajima K, Kosugi S, Ishikawa T, Ajioka Y, Hatakeyama K. Gastrectomy as a secondary surgery for stage IV gastric cancer patients who underwent S-1-based chemotherapy: a multi-institute retrospective study. Gastric Cancer. 2012;15:235-44.

10. Kim SW. The result of conversion surgery in gastric cancer patients with peritoneal seeding. J Gastric Cancer. 2014;14:266-70.

11. Fujitani K, Yang HK, Mizusawa J, Kim YW, Terashima M, Han SU, et al. Gastrectomy plus chemotherapy versus chemotherapy alone for advanced gastric cancer with a single non-curable factor (REGATTA): a phase 3, randomised controlled trial. Lancet Oncol. 2016 (Epub ahead of print).

12. Yoshida K, Yamaguchi K, Okumura N, Tanahashi T, Kodera Y. Is conversion therapy possible in stage IV gastric cancer: the proposal of new biological categories of classification. Gastric Cancer. 2015 (Epub ahead of print). 